\title{
Absorbed Power of Small Children
}

\author{
J. Giacomin Ph.D., M.M.E., B.M.E. \\ Department of Mechanical Engineering \\ The University of Sheffield \\ Mappin Street, Sheffield S1 3JD
}

\section{Tel: 0114-222-7781 Fax: 0114-222-7890 e-mail: j.a.giacomin@sheffield.ac.uk}

\section{Abstract}

Objective: To experimentally measure the seated vertical direction whole-body absorbed power characteristics of small children less than $18 \mathrm{~kg}$ in mass.

Background: Several studies have reported whole-body absorbed power for adult humans, but no data has been published previously for small children.

Methods: Eight children were tested in a laboratory test rig which incorporated safety features which satisfy existing international standards for human testing. Force and acceleration were measured at the point of input to a rigid seat at a sampling rate of $200 \mathrm{~Hz}$, and analysis was performed over the interval from 1.0 to $45.0 \mathrm{~Hz}$. A double normalised (both input acceleration and test subject mass) measure of absorbed power was used.

Results: The vertical whole-body power absorption characteristics of the small children were found to present differences with respect to those of adults. The mean frequency of peak absorption was found to be $7.4 \mathrm{~Hz}$ as opposed to approximately 4.0 to 5.0 for adults. The interval of absorption was found to be from approximately 3 to $16 \mathrm{~Hz}$ and the total double normalised absorbed power was found to be $86 \%$ that of adults.

Conclusions: The differences in dynamic response between small children and adults raise questions regarding the applicability of whole-body vibration guidelines such as ISO-2631 in the case of small children since these guidelines were developed from mechanical and subjective response data of adults. 


\section{Relevance}

Knowledge of the differences in whole-body vibration response between small children and adults is useful towards the design of systems intended for children such as child seats. The child data may also prove useful should absorbed power find application as a screening tool in clinical settings.

Keywords: children, whole-body, vibration, absorbed, power, seat

\section{Introduction}

Numerous research studies have measured the whole-body vibration response of adult humans (Dupuis and Zerlett 1986, Griffin 1990). One response property which has been proposed as a possible metric of the human susceptibility to the physically damaging effects of mechanical vibration has been the absorbed power. The use of absorbed power has origin in the assumption that energy absorbed by the body is energy which can strain and heat internal structures. There has been some debate (Lunström and Holmlund 1998, Mansfield and Griffin 1998) regarding the use of absorbed power, as opposed to frequency weightings derived from subjective responses, as a measure of damage potential. While he clinical interpretation of absorbed power requires further investigation, the measurements themselves remain important descriptions of human biomechanical response.

The origins of the absorbed power approach can be traced to a 1964 study by Weis et. al. which treated the theoretical framework and the possible uses of human driving point mechanical impedance measurements. The authors noted that the two quantities used to determine mechanical impedance also defined the instantaneous power

$$
P(\omega)=F(\omega) V(\omega)
$$

where $P(\omega)$ is the instantaneous power, $F(\omega)$ is the instantaneous force, $V(\omega)$ is the instantaneous velocity and $\omega$ the frequency of oscillation. The expression for the instantaneous power could therefore be rearranged in terms of impedance.

$$
P(\omega)=Z(\omega) V(\omega) V(\omega)=Z(\omega)|V(\omega)|^{2}
$$

Since impedance $Z(\omega)$ is a complex measure the authors suggested inspecting the real and imaginary parts individually 


$$
Z_{\mathrm{Re}}(\omega)=|Z(\omega)| \cos \angle Z(\omega)
$$

and

$$
Z_{\mathrm{Im}}(\omega)=|Z(\omega)| \sin \angle Z(\omega)
$$

The motion of a pure mass element, which does not absorb energy, is characterised by a velocity lag of 90 degrees with respect to the force. Likewise, the motion of a linear spring which is also incapable of energy absorption is characterised by a velocity which leads the force by 90 degrees. The motion of a linear (viscous) damper which dissipates energy is characterised by a velocity and force which are in phase. These observations suggest that only the real component of the power, and thus of a measured impedance, represents energy that is not returned to the vibrating surface from the body. Weis et. al. noted that only the imaginary part of their whole-body data sets was significant at frequencies below $5 \mathrm{~Hz}$, suggesting that the human body acted as a mass below this frequency. The data sets had large values of both the imaginary and the real parts of the impedance in the region from 5 to $15 \mathrm{~Hz}$, and the balance between the two was found to be "complex and highly dependent on sitting posture" for frequencies greater than $15 \mathrm{~Hz}$. The region from 5 to $15 \mathrm{~Hz}$ was therefore singled out as important due to the high transfer of energy into the body.

A 1968 study by Lee and Pradko presented the results of what was described as "extensive testing" of seated human subjects. Tabulated values of impedance modulus, impedance phase angle and absorbed power were presented for the frequency interval from 0 to $12 \mathrm{~Hz}$. Results for all three axis of seated wholebody vibration were presented as well as vertical absorbed power for the feet alone. Lee and Pradko suggested the potential superiority of absorbed power over driving point measurements of mechanical impedance due to absorbed power being a physically interpretable quantity and because, being a scalar, it could be easily summed along different axis.

A 1998 study by Lundström et. al. presented absorbed power data for 15 males and 15 females tested using sinusoidal vibration from 2 to $100 \mathrm{~Hz}$ at root mean square (r.m.s.) amplitudes from 0.5 to $1.4 \mathrm{~m} / \mathrm{s}^{2}$. Absorbed power was found to increase with frequency until 4 to $6 \mathrm{~Hz}$ after which it decreased. The frequency of maximum absorbed power decreased with increasing amplitude of vibration and when changing from an erect to a relaxed posture. The absorbed power was found to increase with the square of the input acceleration amplitude and proportionally with subject mass. To facilitate comparisons the authors mass normalised their data, and the resulting curves were found to be nearly identical to the 1968 Lee and Pradko results. A further point noted by the authors was the difference in the frequency 
dependence of their average absorbed power curves with respect to the frequency weightings of ISO 2631-1 (1997) which are based on subjective response data. The authors suggested that if absorbed power were found to be predictive of damage to biological tissue then the ISO weightings were overestimating the damage potential at frequencies below $6 \mathrm{~Hz}$ while underestimating the damage potential for frequencies greater than $6 \mathrm{~Hz}$.

A 1998 study by Lundström and Holmlund presented absorbed power data for the same group of 15 male and 15 female subjects, but along three axis of vibration. Sinusoidal vibration was used at frequencies from 1 to $80 \mathrm{~Hz}$ and r.m.s. amplitudes from 0.25 to $1.4 \mathrm{~m} / \mathrm{s}^{2}$. Absorbed power was found to increase with frequency up to a peak in the interval from 4 to $6 \mathrm{~Hz}$ for vertical vibration and to increase up to $3 \mathrm{~Hz}$ for fore-and-aft and lateral vibration. Females were found to absorb greater power per kilogram than males. The authors again noted the different conclusions that would be drawn by use of the ISO 2631 frequency weightings as opposed to a line of constant absorbed power.

A 1998 study by Mansfield and Griffin measured seated absorbed power for 12 male subjects using random excitation in the interval from 0.2 to $20 \mathrm{~Hz}$ at r.m.s. acceleration amplitudes from 0.25 to $2.5 \mathrm{~m} / \mathrm{s}^{2}$. The authors noted that it was convenient to determine the absorbed power from the cross spectrum between the velocity and force as

$$
P_{a b s}(\omega)=P_{\mathrm{Re}}(\omega)=\left|G_{V F}(\omega)\right| \cos (\theta(\omega))
$$

(5)

where $\left|G_{V F}(\omega)\right|$ is the modulus and $\theta(\omega)$ the phase angle of the cross spectrum between the driving point input velocity and output force. Since absorbed power increases with the square of the acceleration the authors chose acceleration to normalise their data, compensating in this way any variations in the tests. The mean frequency of maximum absorption was found to be $5 \mathrm{~Hz}$. This frequency was lower, and the absorption greater, at high vibration amplitudes. The authors compared their results to those of both Lee and Pradko (1968) and Lundström et. al. (1998) and concluded that there was remarkable similarity between the data sets. Like Lundström et. al. they compared their absorbed power curves to the ISO 2631 weightings. Unlike Lundström et. al., they concluded that "the absorbed power is unlikely to yield good general predictions of the discomfort or risks of injury from whole-body vertical vibration".

A 2001 study by Mansfield et. al. measured vertical apparent mass and absorbed power for 24 subjects exposed to 15 vibration conditions. The input acceleration signals were of 5 types and were scaled to three r.m.s. amplitudes: $0.5,1.0$ and $1.5 \mathrm{~m} / \mathrm{s}^{2}$. The shape of the absorbed power curves was found to change little with excitation type or amplitude, but the frequency value of the absorbed power peak was found to drop with increasing input amplitude. For individual subjects the total absorbed power, calculated as the 
integral of the absorbed power curve from 3 to $20 \mathrm{~Hz}$, showed statistically significant increases for shock signals with respect to continuous vibration.

An important information that has not yet been presented in the research literature is the absorbed power characteristics of user groups other than healthy adults, such as small children. The whole-body vibrational behaviour of small children is of relevance towards the design of systems such as child safety seats. Vibrational measurements for child seats were first reported by Giacomin (2000 and 2003) who measured acceleration at the interface between child and child seat in automobiles while driving. The same author has also experimentally measured the whole-body apparent mass of a group of eight small children (Giacomin 2004). This paper describes, instead, experimental measurements of absorbed power performed for a group of eight small children of the size and age range appropriate to stage 0\&1 safety seats (less than $18 \mathrm{~kg}$ in mass and less than 4 years of age).

\section{Methods}

\subsection{Test Facility}

The tests were performed by means of a laboratory facility designed for use with small children. The subjects were seated in a rigid aluminium basket whose five primary dimensions (see Figure 1) were a lower section length $\left(L_{1}\right)$ of $300 \mathrm{~mm}$, a backrest section length $\left(L_{2}\right)$ of $450 \mathrm{~mm}$, a width of $280 \mathrm{~mm}$, an included angle $F$ of 90 degrees and an angle of 20 degrees with respect to the horizontal $(T)$. The basket was fabricated of $1.5 \mathrm{~mm}$ thick aluminium sheet and was welded to an aluminium base section. The basket unit had a mass of 3.0 kilograms and a first natural frequency greater than $170 \mathrm{~Hz}$. It was equipped with restraining belts from a commercial child seat, which were fastened in the same manner as with the commercial product.

\section{[INSERT FIGURE 1 HERE] [INSERT FIGURE 2 HERE]}

The seat basket was mounted by means of three load cells to a $760 \mathrm{~mm}$ long by $405 \mathrm{~mm}$ wide aluminium platform which was suspended by springs and linear bearings. Stiffness adjustment permitted the first natural frequency of the system composed of child, sitting basket and platform to be kept below $1 \mathrm{~Hz}$. The system was vibrated by means of a Ling Dynamic Systems (LDS) V406 permanent magnet shaker and accompanying PA100E power amplifier. Vibration measurement and control were achieved using an LMS Endurance Monitor (EMON) system coupled to a DIFA Systems SCADAS II electronic unit. The EMON software permitted the fixing of numerical limits for the test acceleration and force, which were set to 2.0 $\mathrm{m} / \mathrm{s}^{2}$ peak acceleration and $100 \mathrm{~N}$ peak transmitted force. A DIFA Systems Shutdown Control Unit was also used which incorporated a manual emergency shutdown button. 
Acceleration was measured using an ENTRAN EGAS-FT-25 accelerometer fixed to the seat basket. The maximum acceleration rating of the sensor was $25 \mathrm{~g}$, it had a sensitivity of $3.66 \mathrm{mV} / \mathrm{g}$ and had a natural frequency of $730 \mathrm{~Hz}$. The force transmitted to the rigid seat was measured using three load cells placed at the apexes of a triangle. The three sensors were ENTRAN type ELH-TC11-500s which were rated as linear to $500 \mathrm{~N}$ and had a sensitivity of $0.16 \mathrm{mV} / \mathrm{N}$. Figure 2 presents a schematic diagram of the test facility.

\subsection{Test Vibration}

Band limited random excitation was chosen in order to achieve short tests. A band-limited Gaussian white noise acceleration signal was defined over the interval from 1 to $50 \mathrm{~Hz}$ using a sampling rate of $200 \mathrm{~Hz}$. Preliminary runs showed that the input r.m.s. acceleration at the platform needed to be greater than 0.6 $\mathrm{m} / \mathrm{s}^{2}$ to overcome friction in the bearings and to obtain a good signal-to-noise ratio. Two drive time histories were therefore defined by scaling a base signal to r.m.s. voltage levels of $1400 \mathrm{mV}$ and $1800 \mathrm{mV}$ corresponding to actuated r.m.s. acceleration levels of 0.8 and $1.2 \mathrm{~m} / \mathrm{s}^{2}$ at the platform and seat basket.

In order to determine the time duration of the vibration exposure the guidelines of British Standards Institution BS 7085 Safety aspects of experiments in which people are exposed to mechanical vibration and shock were adopted. BS 7085 specifies that vibration exposure should be quantified in terms of the Vibration Dose Value (VDV)

$$
V D V=\left[\int_{0}^{T} a^{4}(t) d t\right]^{1 / 4}
$$

where $a(t)$ is the frequency weighted acceleration in $\mathrm{m} / \mathrm{s}^{2}$ and $T$ is the period of exposure in seconds. BS7085 suggests the use of frequency weighting curve $W_{b}$ when quantifying the effects of vertical direction vibration input to the buttocks through a seat, and recommends a maximum frequency weighted VDV value of $15 \mathrm{~m} / \mathrm{s}^{1.75}$ as the exposure limit which should not be exceeded without the presence of a medical officer. Applying the $W_{b}$ frequency weighting to the platform acceleration resulted in weighted r.m.s. values of $0.52 \mathrm{~m} / \mathrm{s}^{2}$ for the $1400 \mathrm{mV}$ signal and $0.75 \mathrm{~m} / \mathrm{s}^{2}$ for the $1800 \mathrm{mV}$ signal. Time duration for both signals was chosen to be 2 minutes, producing VDV values of 10.0 and $14.0 \mathrm{~m} / \mathrm{s}^{1.75}$ respectively.

\subsection{Test Calibration}

The facility was calibrated using rigid masses. Four steel blocks were used of mass 3.8, 7.8 and 15.8 kilograms respectively. An assembly consisting of two threaded rods and two cross members weighting a 
total of $0.2 \mathrm{~kg}$ was used to fasten the masses to the rigid seat. Band-limited Gaussian random white noise voltage signals were input to the bench through a Finite Impulse Response Filter (FIR) filter designed to compensate the low force output of the V406 at frequencies below $20 \mathrm{~Hz}$. Calibration was performed at levels from $1000 \mathrm{mV}$ to $1800 \mathrm{mV}$ in steps of $200 \mathrm{mV}$, with mass loadings of $0 \mathrm{~kg}, 4 \mathrm{~kg}, 8 \mathrm{~kg}$ and $16 \mathrm{~kg}$. Since a rigid mass cannot absorb power, the deviation from zero was verified across the calibration conditions and found to be less than $0.0001(\mathrm{Nm} / \mathrm{s}) / \mathrm{Hz}$. The apparent mass modulus curves obtained with the calibration blocks contained errors of less than $2 \%$ in the interval from 2.0 to $35.0 \mathrm{~Hz}$ and less than $10 \%$ over the range from 1.0 to $42.0 \mathrm{~Hz}$. The coherence function was found to be approximately 1.0 over the frequency range from 1 to $45 \mathrm{~Hz}$. The first resonance frequency of the unit consisting of seat basket and aluminium platform was found to be always greater than $50 \mathrm{~Hz}$ under all calibration loading conditions.

\subsection{Test Protocol}

Eight children were tested whose ages ranged from 3 to 23 months with a mean value of 11.8. Their weight ranged from 5.0 to $12.4 \mathrm{~kg}$ with a mean value of $9.5 \mathrm{~kg}$ and their heights ranged from 50 to $86 \mathrm{~cm}$ with a mean value of 73.4 as shown in Table 1. Each child was brought to the test facility by the consenting parent or parents who were all staff or students of Sheffield University.

\section{[INSERT TABLE 1 HERE]}

Upon arrival in the laboratory the parents were explained the research objectives and methods. A verbal description of the safety features was provided, and the type and level of acceleration signal used was specified. Acceleration levels commonly found in automobiles were also provided as a reference for comparison. The parent or parents were next asked to read and sign a participation form, after which child supine length was measured on a table and weight measured using a scale. Time was then dedicated to play and familiarisation such that the child could become acquainted with, and comfortable in, the laboratory environment. Once the child appeared happy, he or she was placed in the test seat by a parent and the belt straps were adjusted by the researcher. A cot mobile was then activated and reading to the child from children's books was initiated.

An automated EMON playlist was then activated. A complete test sequence consisted of a 2 minute exposure using the $1400 \mathrm{mV}$ signal followed by the saving of all data, followed by the 2 minute $1800 \mathrm{mV}$ signal and the saving of all $1800 \mathrm{mV}$ data. A maximum of two sequences were performed for any child on a single day. The EMON playlist could be interrupted at any point through operator manual override. The test protocol was terminated in any case in which a child demonstrated unhappiness with, or fear of, the test environment. The full test protocol was externally reviewed and found to meet the University of Sheffield guidelines for good research practice. No payments or other incentives were offered to either the parents 
or child and no deception was practiced regarding any aspect of the research. A detailed description of the ethical and safety features of the facility and protocol is provided by Giacomin (2004).

\section{Results}

Acceleration and reaction force were acquired as time histories sampled at $200 \mathrm{~Hz}$. After acquisition the acceleration time histories were single integrated in the frequency domain and a $5^{\text {th }}$ order polynomial was applied in the time domain to reduce low frequency trends. The cross spectrum between velocity and force was then determined using a Hanning window with a block size of 512 points and an overlap of $97 \%$, which lead to a spectral resolution of $0.39 \mathrm{~Hz}$ and a total of 1541 averages for each 2 minute test exposure.

Absorbed power was determined from the cross spectrum between the velocity and the force as defined by equation 5 , and plotted in spectral density form in units of $(\mathrm{Nm} / \mathrm{s}) / \mathrm{Hz}$. Since absorbed power has been shown to increase with the square of the input acceleration, the data was also acceleration normalised in the manner defined by Lee and Pradko (1968) and Mansfield and Griffin (1998). In this form the absorbed power spectral density is divided by the square of the acceleration power spectral density leading to units of $\mathrm{Ns}^{3} / \mathrm{m}$. Lunström et. al. (1998) have further noted that the power absorbed by humans increases in proportion to body mass, leading them to normalise their data by the test subject body mass in kilograms. In this case the results were presented in units of Watts per kilogram in spectrum form and units of $\mathrm{W} / \mathrm{kgHz}$ in spectral density form. Consideration of the effects of both test signal acceleration and test subject mass suggested the usefulness of defining a double normalised measure having units of $\mathrm{Ns}^{3} / \mathrm{kgm}$. This measure, presented here for the first time, was calculated from the child absorbed power results and used to compare the child data to the results from previous studies involving adults.

Figure 3 presents the absorbed power spectral density in units of $(\mathrm{Nm} / \mathrm{s}) / \mathrm{Hz}$ for the eight child subjects at the r.m.s. test acceleration levels of 0.8 and $1.2 \mathrm{~m} / \mathrm{s}^{2}$. As expected, the absorbed power increased with both acceleration amplitude and subject mass. At $5.2 \mathrm{~kg}$ the smallest child (ju) absorbed the least energy while at $12.4 \mathrm{~kg}$ the largest (sa) absorbed the most. The frequency of peak power absorption suggested a single whole-body response resonance at approximately $7.4 \mathrm{~Hz}$. All absorbed power measurements were characterised by a noisy appearance, containing multiple peaks which upon first inspection might suggest multiple body resonances. These variations in the absorbed power were due, however, to small variations in the input acceleration spectra. Normalising each frequency line by the input acceleration spectra reduced this phenomena and lead to normalised absorbed power curves which were smoother in appearance. All acceleration normalised absorbed power curves are presented in Figure 4. The curves confirm a single resonance and also confirm a relatively high level of linearity due to the similarity of the data from the $1400 \mathrm{mv}$ and $1800 \mathrm{mv}$ tests. Changes in the modulus peak for subjects le, ma, and mo show some migration towards a lower frequency, while the changes for subject ju suggest the opposite. 
From only the data of the eight subjects tested in the current study it is not possible to confirm or reject a hypothesis of nonlinear softening system behaviour of the type often found for adult humans. It is nevertheless of interest to note that the heaviest child (sa) showed possible evidence of softening system behaviour while the lightest (ju) was the only subject possibly showing the opposite.

\section{[INSERT FIGURE 3 HERE] [INSERT FIGURE 4 HERE]}

\section{[INSERT FIGURE 5 HERE] [INSERT FIGURE 6 HERE]}

\section{[INSERT FIGURE 7 HERE] [INSERT TABLE 3 HERE]}

The total power absorbed, which is obtained by integration, was also determined. The mean total absorbed power for the group of children tested at $1.2 \mathrm{~m} / \mathrm{s}^{2}$ can be compared to that of the adults who were tested at $1.0 \mathrm{~m} / \mathrm{s}^{2}$ by Mansfield and Griffin (1998). At $0.0156 \mathrm{Ns}^{2} / \mathrm{m}$ for the children and $0.1289 \mathrm{Ns} / \mathrm{m}$ for the adults the total power absorption for the children was approximately $12 \%$ that of adults. When determining the total absorbed power in double normalised form, however, the two values are found to be closer at 0.0016 $\mathrm{Ns}^{2} / \mathrm{kgm}$ for the children and $0.0018 \mathrm{Ns}^{2} / \mathrm{kgm}$ for the adults, suggesting that child absorption is approximately $86 \%$ that of adults when considered on a per-kilogram basis. Small children may therefore be slightly less efficient absorbers of vibrational energy than adults. It must be considered, however, that the differences may also be attributable in part to differences in sitting posture since the small children were tested with back support while the adults had no back support.

\section{Discussion}

A first point for consideration is the similarity between the absorbed power curves obtained for the eight children. The uniformity suggests the usefulness of the current results despite the small sample size used. This finding may serve as a source of encouragement for the designers of systems such as child safety seats since the variation in the biomechanical response is sufficiently small as to suggest the usefulness of design studies performed, of necessity, with small samples.

A second consideration is that he child absorbed power curves differed from those of adult humans. Seven of the eight children were characterised by absorbed power curves which contained only a single resonance peak. Only the largest child, subject SA, exhibited a dynamic response which might suggest two whole-body resonances as found for seated adult humans by Lundström and Holmlund (1998), Lundström et. al. (1998), Mansfield and Griffin (1998) and Mansfield et. al. (2001). Further, the mean frequency of peak power absorption was found to be approximately $7.4 \mathrm{~Hz}$ for the small children as opposed to values in the range from 4.0 to $5.0 \mathrm{~Hz}$ for adults. Differences were also found in the total 
double normalised absorbed power, with mean child absorption being approximately $86 \%$ that of adults. A possible implication of these differences is that the use of whole-body vibration guidelines such $\boldsymbol{\infty}$ ISO2631, which were developed based on data from adult humans, may prove inappropriate in the case of small children under $18 \mathrm{~kg}$ in mass. A possible confounding factor to this conclusion is that sitting posture may also play a role in the observed differences. The children were tested in the current study using a rigid seat which provides some back support, whereas all absorbed power tests reported in the literature for adult humans have used unsupported postures (no backrest). Until such time as backrest supported absorbed power data is available for adults for comparison, caution would seem appropriate when applying existing whole-body vibration guidelines to child subjects.

In the current study a double normalised representation of absorbed power was defined. The motivation behind the approach is that absorbed power has been shown in the research literature to grow as a function of both the input acceleration amplitude and body mass. Normalising by both quantities provides a representation which appears useful for comparing the dynamic properties of humans of different size. Double normalised absorbed power would be expected to offer advantages over existing methods if absorbed power were used as a screening tool in clinical settings.

\section{References}

British Standards Institution 1989. BS 7085 Safety aspects of experiments in which people are exposed to mechanical vibration and shock. British Standards Institution, London.

Dupius, H. and Zerlett, G. 1986. The effects of whole-body vibration. Springer-Verlag, Berlin.

Giacomin, J. 2004, Apparent mass of small children: experimental measurements, Ergonomics, 47 (13), 1454-1474.

Giacomin, J. and Gallo, S. 2003, In-vehicle vibration study of child safety seats, Ergonomics, 46 (15), 1500-1512.

Giacomin, J. 2000. Some observations regarding the vibrational environment in child safety seats. Applied Ergonomics, 31, 207-215.

Griffin, M.J. 1990. Handbook of human vibration. Academic Press, London.

Holmlund, P. 1999. Absorbed power and mechanical impedance of the seated human measured within a real vehicle environment compared with single axis laboratory data. Journal of Low Frequency Noise, Vibration and Active Control, 8 (3), 97-110. 
International Organisation for Standardisation 1997. ISO 2631-1 (E) Mechanical vibration and shock evaluation of human exposure to whole-body vibration. International Organisation for Standardisation, Geneva.

Lee, R.A. and Pradko, F. 1968. Analytical analysis of human vibration. SAE paper 680091 . Society of Automotive Engineers, Warrandale, PA.

Lundström, R. and Holmlund, P. 1998. Absorption of energy during whole-body vibration exposure. Journal of Sound and Vibration, 215 (4), 789-799.

Lundström, R., Holmlund, P. and Lindberg, L. 1998. Absorption of energy during vertical whole-body vibration exposure. Journal of Biomechanics, 31, 317-326.

Mansfield, N.J. and Griffin, M.J. 1998. Effect of magnitude of vertical whole-body vibration on absorbed power for the seated human body. Journal of Sound and Vibration, 215 (4), 813-825.

Mansfield, N.J., Holmlund, P. and Lundström, R. 2001. Apparent mass and absorbed power during exposure to whole-body vibration and repeated shocks. Journal of Sound and Vibration, 248 (3), 427-440.

Weis, E.B., Clarke, N.P., Brinkley, J.W. and Martin, P.J. 1964. Mechanical impedance as a tool in research on human response to acceleration. Aerospace Medicine, 35, 945-950. 


\begin{tabular}{|c|c|c|c|c|}
\hline Child & Age (months) & Mass (kg) & Height (cm) & Sex (M/F) \\
\hline al & 10.0 & 9.4 & 76.0 & $\mathrm{M}$ \\
\hline $\mathrm{im}$ & 15.0 & 11.0 & 86.0 & $\mathrm{M}$ \\
\hline $\mathrm{ja}$ & 13.0 & 11.4 & 80.0 & $\mathrm{M}$ \\
\hline $\mathrm{ju}$ & 3.0 & 5.2 & 50.0 & $\mathrm{~F}$ \\
\hline $\mathrm{le}$ & 8.5 & 9.4 & 73.0 & $\mathrm{M}$ \\
\hline $\mathrm{ma}$ & 7.0 & 8.0 & 66.0 & $\mathrm{~F}$ \\
\hline $\mathrm{mo}$ & 14.5 & 10.2 & 71.0 & $\mathrm{~F}$ \\
\hline sa & 23.0 & 12.4 & 85.0 & $\mathrm{~F}$ \\
\hline Mean & $\mathbf{1 1 . 8}$ & $\mathbf{9 . 6}$ & $\mathbf{7 3 . 4}$ & - \\
\hline Std. & $\mathbf{6 . 1}$ & $\mathbf{2 . 2}$ & $\mathbf{1 1 . 7}$ & - \\
\hline
\end{tabular}

Table 1 Child test subject characteristics. 


\begin{tabular}{|c|c|c|}
\hline Frequency $[\mathrm{Hz}]$ & $\begin{array}{l}\text { Acceleration normalised absorbed } \\
\text { power }\left[\mathrm{Ns}^{3} / \mathrm{m}\right]\end{array}$ & $\begin{array}{c}\text { Double normalised absorbed } \\
\text { power }\left(\mathrm{Ns}^{3} / \mathrm{kgm}\right)\end{array}$ \\
\hline 1.95 & 0.0164 & 0.0017 \\
\hline 2.34 & 0.0238 & 0.0025 \\
\hline 2.73 & 0.0265 & 0.0028 \\
\hline 3.12 & 0.0369 & 0.0038 \\
\hline 3.51 & 0.0530 & 0.0055 \\
\hline 3.90 & 0.0686 & 0.0072 \\
\hline 4.29 & 0.0851 & 0.0089 \\
\hline 4.68 & 0.0962 & 0.0100 \\
\hline 5.07 & 0.1191 & 0.0124 \\
\hline 5.46 & 0.1462 & 0.0152 \\
\hline 5.85 & 0.1656 & 0.0172 \\
\hline 6.25 & 0.1850 & 0.0193 \\
\hline 6.64 & 0.1976 & 0.0206 \\
\hline 7.03 & 0.2078 & 0.0216 \\
\hline 7.42 & 0.2171 & 0.0226 \\
\hline 7.81 & 0.2131 & 0.0222 \\
\hline 8.20 & 0.2075 & 0.0216 \\
\hline 8.59 & 0.1977 & 0.0206 \\
\hline 8.98 & 0.1866 & 0.0194 \\
\hline 9.37 & 0.1709 & 0.0178 \\
\hline 9.76 & 0.1634 & 0.0170 \\
\hline 10.15 & 0.1475 & 0.0154 \\
\hline 10.54 & 0.1341 & 0.0140 \\
\hline 10.93 & 0.1213 & 0.0126 \\
\hline 11.32 & 0.1104 & 0.0115 \\
\hline 11.71 & 0.0997 & 0.0104 \\
\hline 12.10 & 0.0923 & 0.0096 \\
\hline 12.50 & 0.0840 & 0.0087 \\
\hline 12.89 & 0.0781 & 0.0081 \\
\hline 13.28 & 0.0732 & 0.0076 \\
\hline 13.67 & 0.0671 & 0.0070 \\
\hline 14.06 & 0.0613 & 0.0064 \\
\hline 14.45 & 0.0559 & 0.0058 \\
\hline 14.84 & 0.0518 & 0.0054 \\
\hline 15.23 & 0.0484 & 0.0050 \\
\hline 15.62 & 0.0445 & 0.0046 \\
\hline 16.01 & 0.0424 & 0.0044 \\
\hline 16.40 & 0.0402 & 0.0042 \\
\hline 16.79 & 0.0379 & 0.0039 \\
\hline 17.18 & 0.0354 & 0.0037 \\
\hline 17.57 & 0.0332 & 0.0035 \\
\hline 17.96 & 0.0310 & 0.0032 \\
\hline 18.35 & 0.0289 & 0.0030 \\
\hline 18.75 & 0.0276 & 0.0029 \\
\hline 19.14 & 0.0261 & 0.0027 \\
\hline 19.53 & 0.0250 & 0.0026 \\
\hline 19.92 & 0.0236 & 0.0025 \\
\hline 20.31 & 0.0223 & 0.0023 \\
\hline 20.70 & 0.0212 & 0.0022 \\
\hline 21.09 & 0.0206 & 0.0021 \\
\hline 21.48 & 0.0201 & 0.0021 \\
\hline 21.87 & 0.0193 & 0.0020 \\
\hline 22.26 & 0.0184 & 0.0019 \\
\hline 22.65 & 0.0178 & 0.0019 \\
\hline 23.04 & 0.0173 & 0.0018 \\
\hline 23.43 & 0.0167 & 0.0017 \\
\hline 23.82 & 0.0162 & 0.0017 \\
\hline 24.21 & 0.0158 & 0.0016 \\
\hline 24.60 & 0.0151 & 0.0016 \\
\hline 25.00 & 0.0143 & 0.0015 \\
\hline
\end{tabular}

Table 2 Mean absorbed power values for the group of eight children tested at $1.2 \mathrm{~m} / \mathrm{s}^{2}$ in both the acceleration normalised $\left(\mathrm{Ns}^{3} / \mathrm{m}\right)$ and double normalised $\left(\mathrm{Ns}^{3} / \mathrm{kgm}\right)$ forms. 

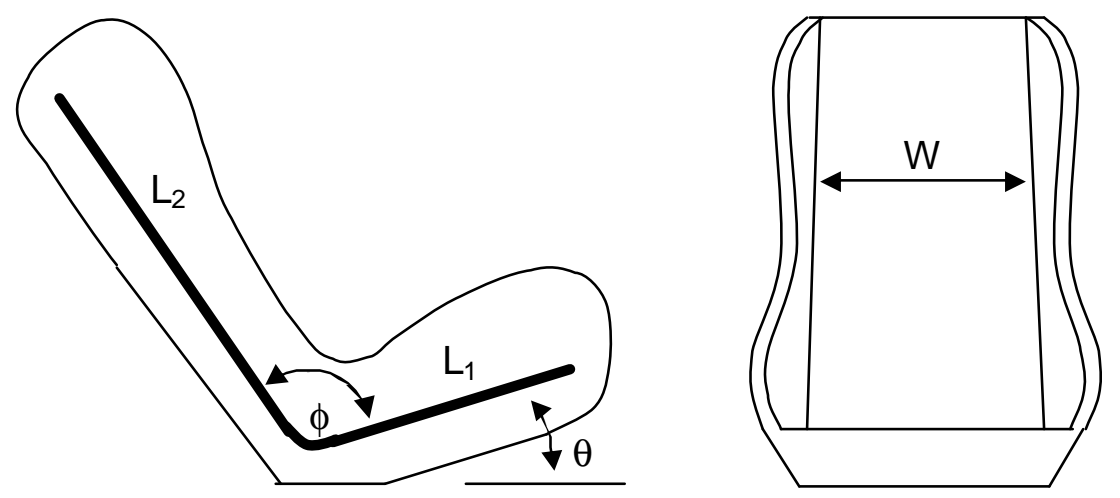

Figure 1 Parameters defining the sitting posture of the test facility. 


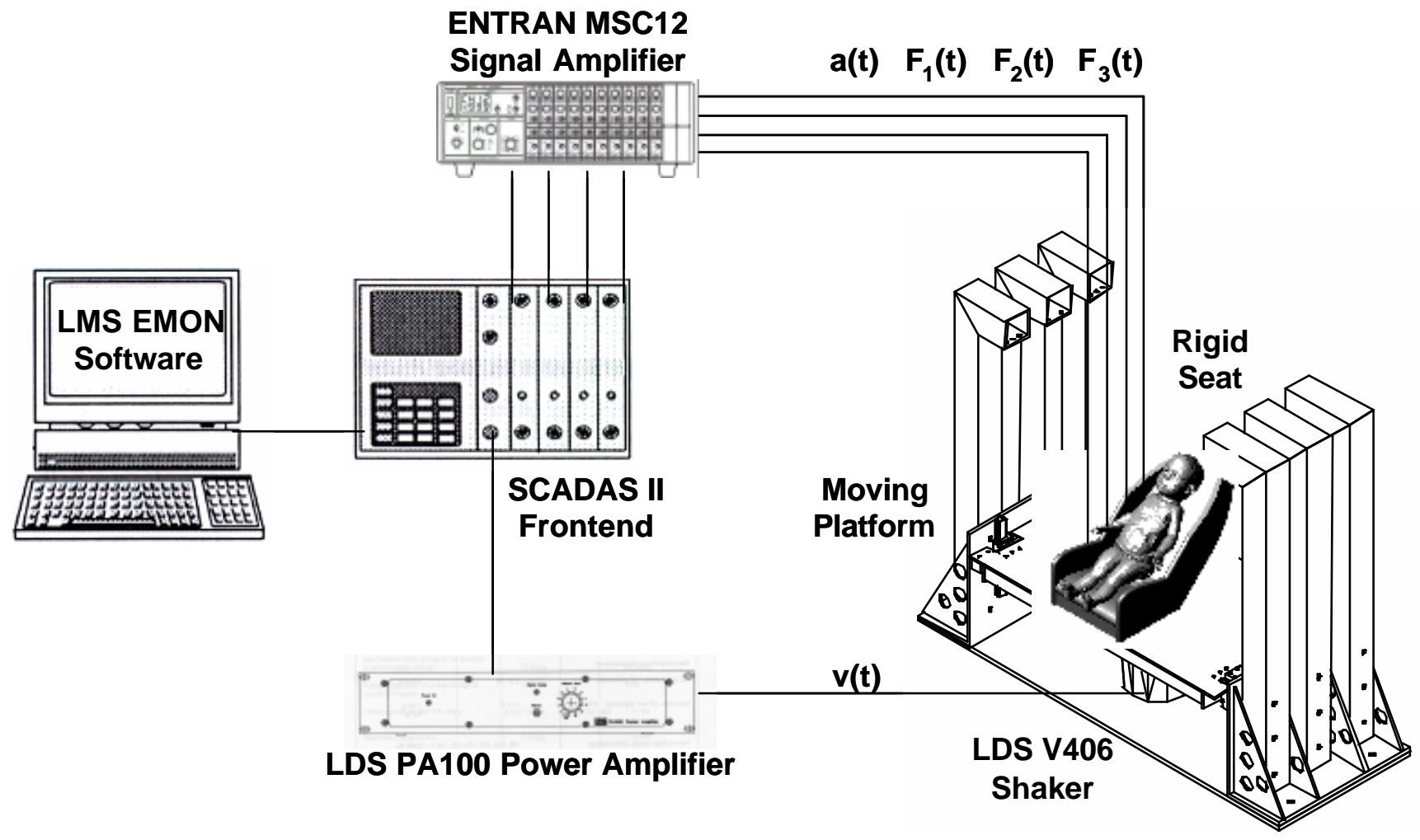

Figure 2 Schematic diagram of the child vibration testing facility. 

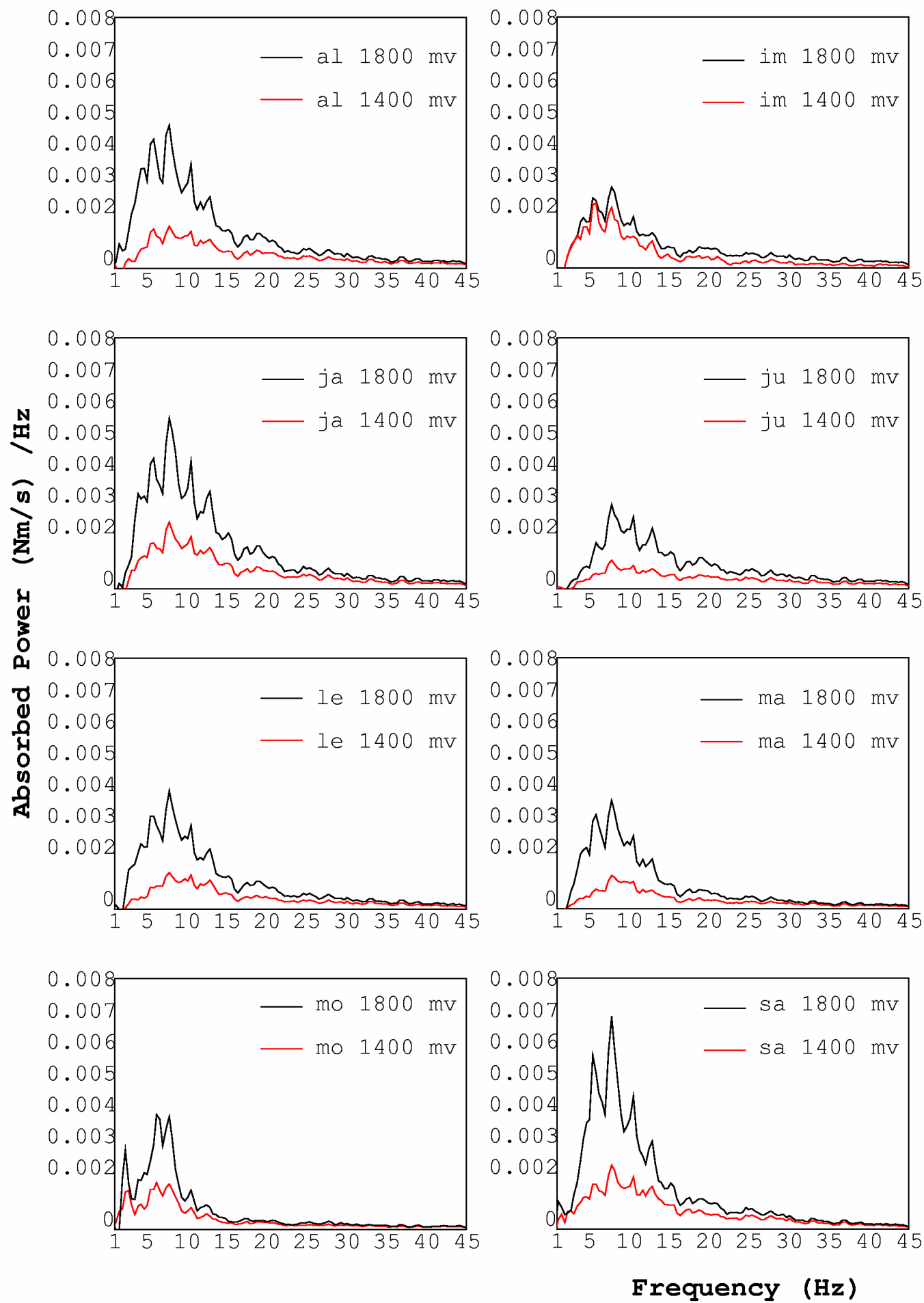

Figure 3 Absorbed power spectral density of eight children tested at r.m.s. acceleration levels of $0.8 \mathrm{~m} / \mathrm{s}^{2}(1400 \mathrm{mV})$ and $1.2 \mathrm{~m} / \mathrm{s}^{2}(1800 \mathrm{mV})$. 

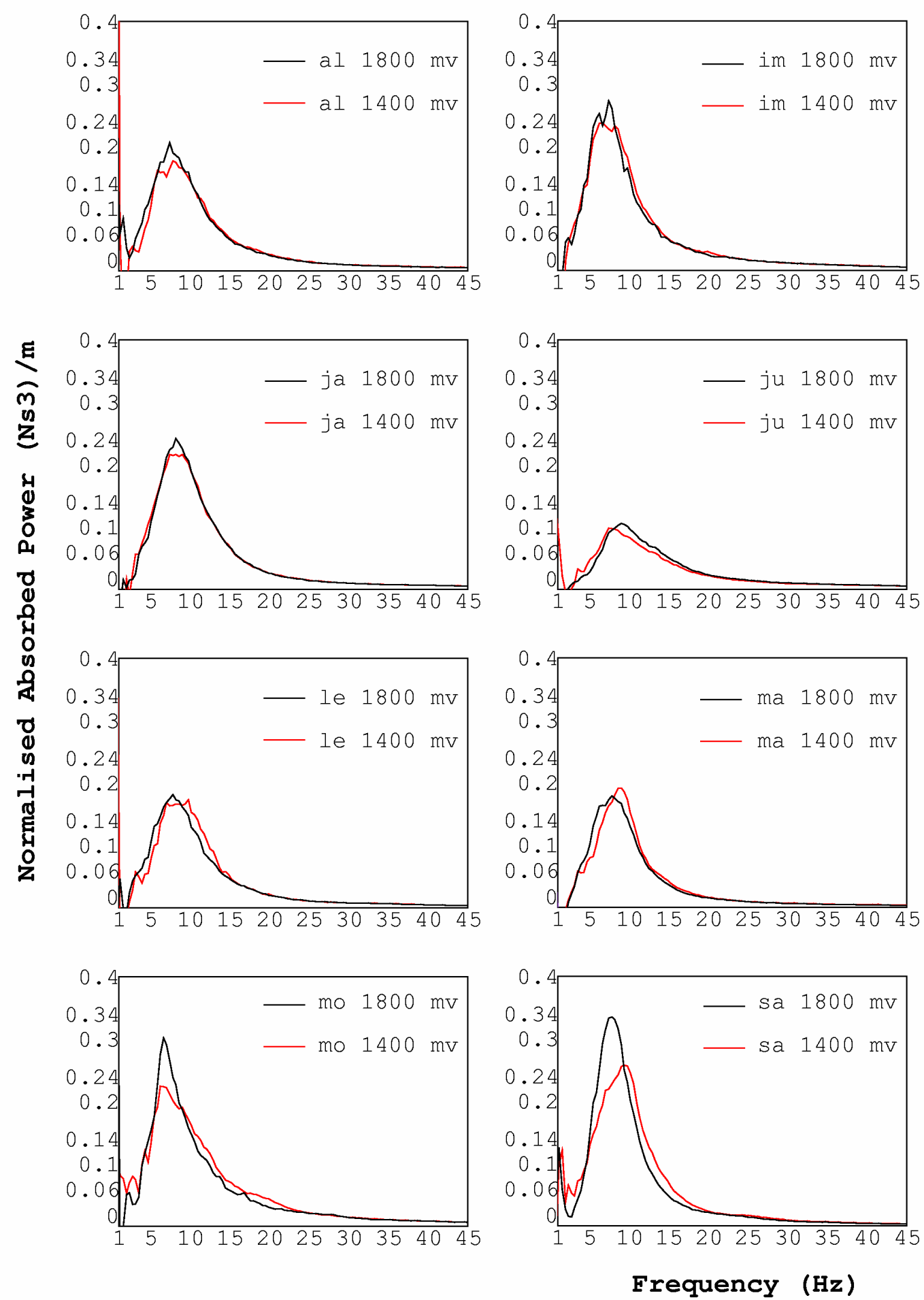

Figure 4 Normalised absorbed power of eight children tested at r.m.s. acceleration levels of $0.8 \mathrm{~m} / \mathrm{s}^{2}(1400 \mathrm{mV})$ and $1.2 \mathrm{~m} / \mathrm{s}^{2}(1800 \mathrm{mV})$. 


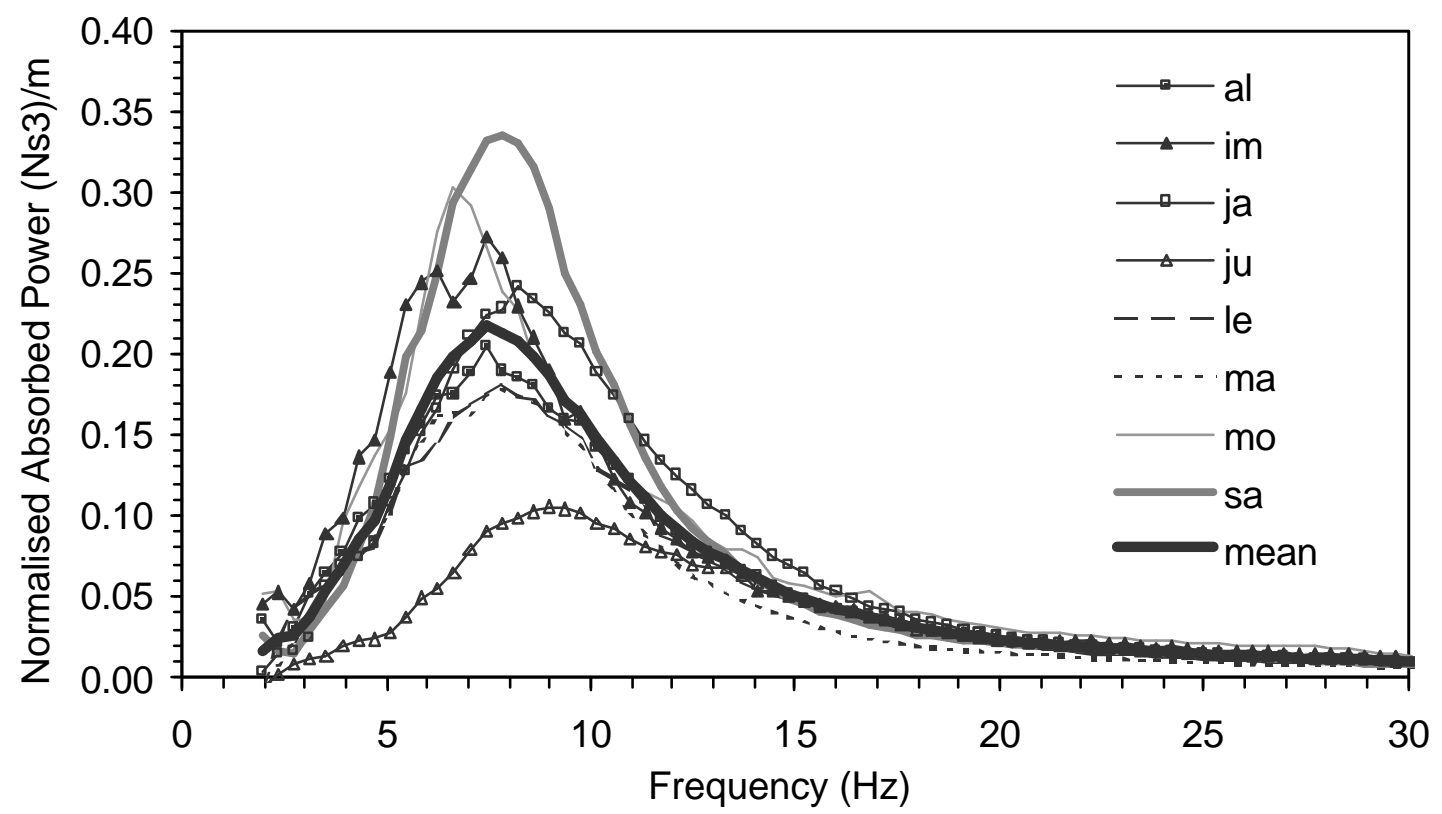

Figure 5 Individual and group mean acceleration normalised absorbed power for eight children tested at $1.2 \mathrm{~m} / \mathrm{s}^{2}$ (1800 $\mathrm{mV}$ test signal). 


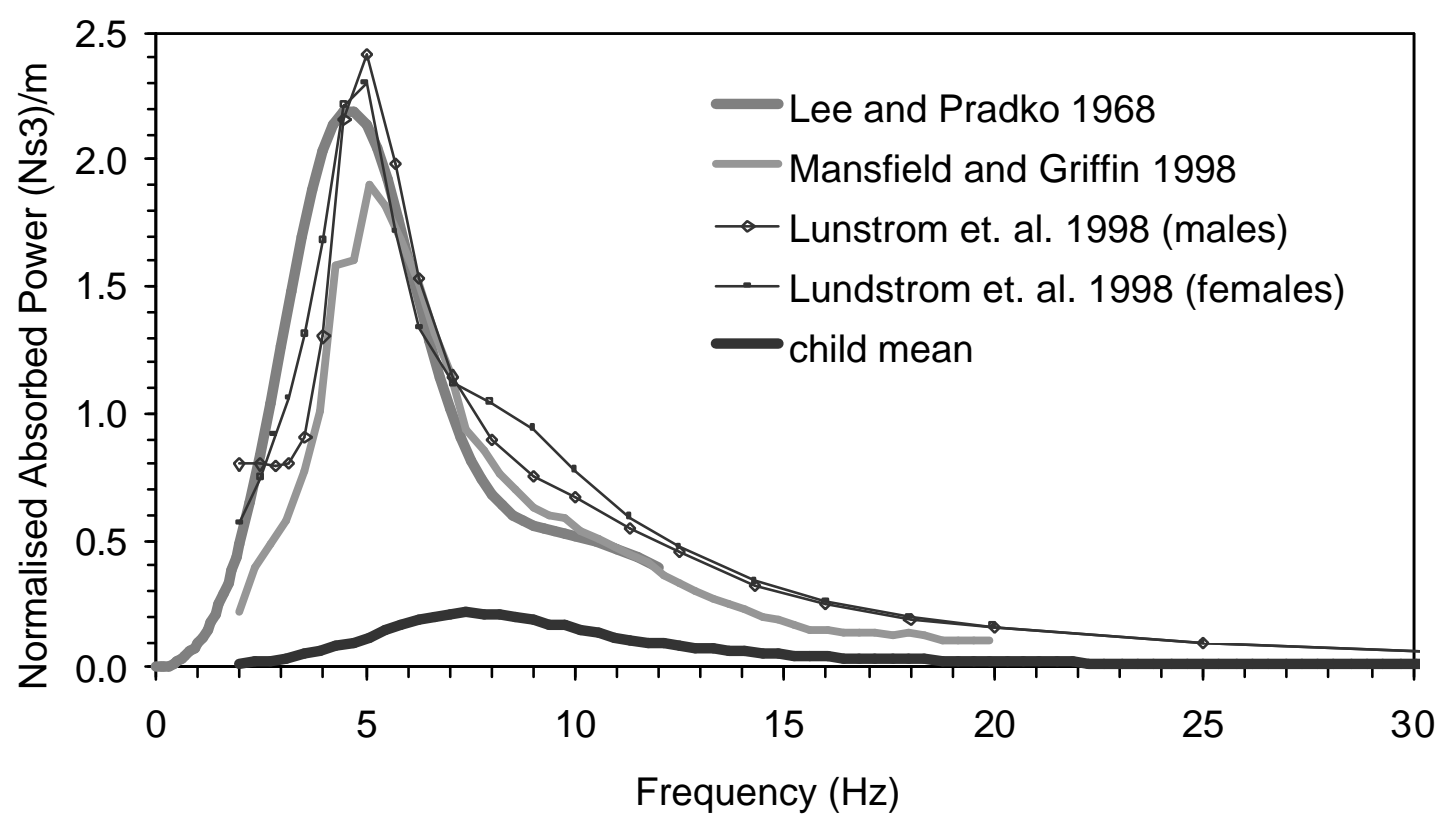

Figure 6 Comparison between the acceleration normalised absorbed power determined in the current study for children tested at $1.2 \mathrm{~m} / \mathrm{s}^{2}$ and results reported in the literature for adults. 


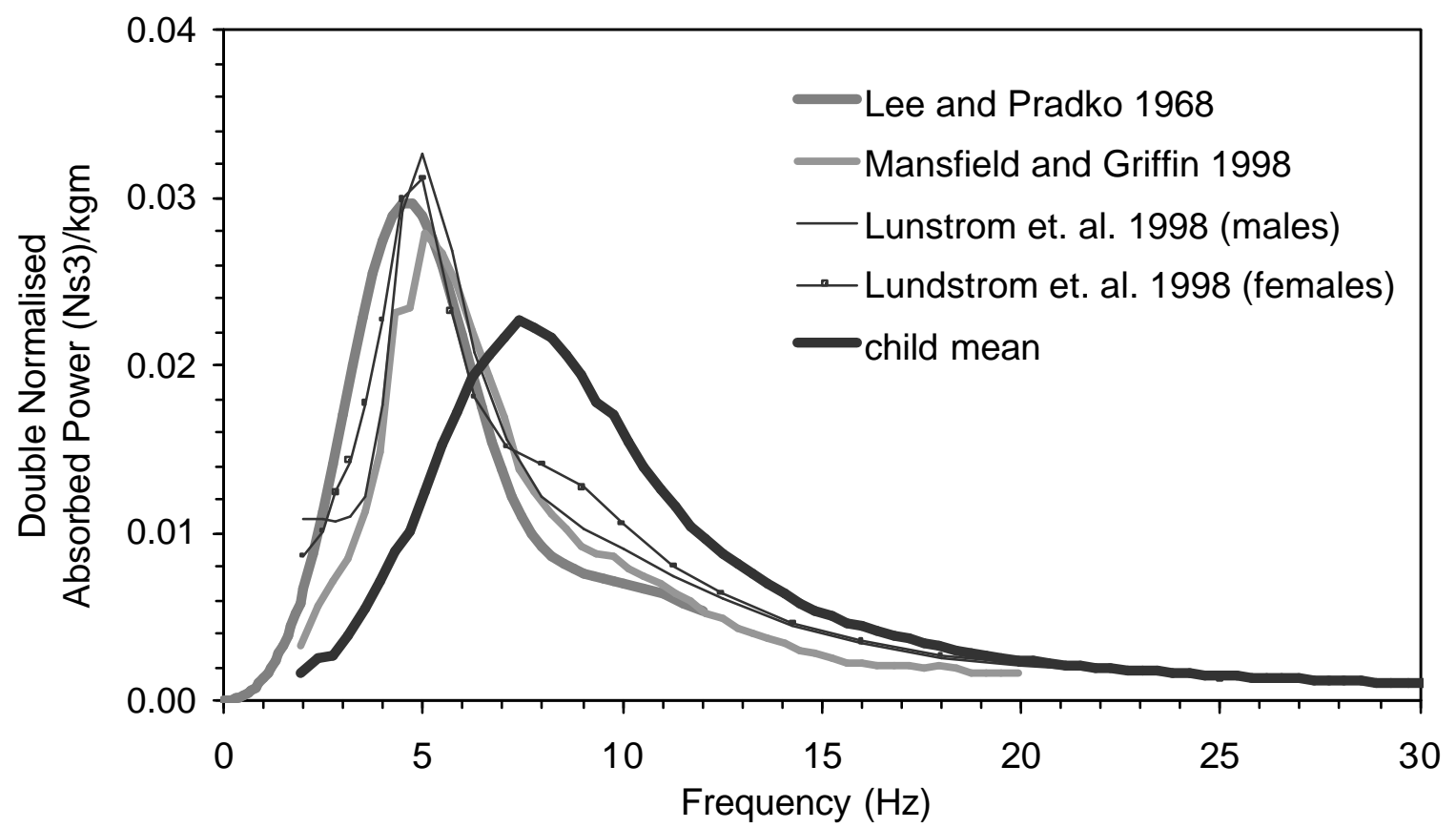

Figure 7 Comparison between the double normalised absorbed power determined in the current study for children tested at $1.2 \mathrm{~m} / \mathrm{s}^{2}$ and results reported in the literature for adults. 